\title{
An approach of demonstrating network structure
}

\author{
Liehuang Zhu ${ }^{1}$ Zhichao Zhou ${ }^{1}$ Zhansheng Ma $^{2}$ \\ ${ }^{1}$ School of Computer Science and Technology, Beijing Institute of Technology \\ ${ }^{2}$ Huihua College of Hebei Normal University
}

\begin{abstract}
In network management, computer-aided technology is introduced to fit the large network. Demonstrating the network topologic structure to managers vividly will help greatly. However, the network graphic demonstration is very difficult. Much as it costs, it lacks methods to form an efficient solution for a long time. To overcome this problem, in this paper, we propose an approach for demonstrating the network structure. Shortest tree could fix the frame of the network structure demonstrating, and implements many models to optimize the graph for network demonstration, until a satisfactory result is obtained. Compared with other traditional approaches, our approach shows significant advantages in both effectiveness and efficiency.
\end{abstract}

Keywords: network structure; demonstration; models

\section{Introduction}

Nowadays more and more individual networks appear to implement high quality or high demand services and applications. Almost all of the network structures have become larger and more complex, with IT developing at such a high speed. Under these considerations, big network management is a difficult work [1], because of the scale of the network and the potential changes in the network structure. Manual handling may be a good idea, be- cause of lacking of managing strategies suit for all kinds of network automatically. Since it is a difficult job, we need help from software; computer aided network management (CANM) will work [2]. Using CANM, people should know all important things about network. So, how to give network manager the information rapidly is an urgent problem to deal with in CANM.

There is no way better than to give network manager a whole understanding on his network. Presenting network structure to the manager will be a good method to give him a whole understanding which can help him to make a reasonable decision as soon as possible [3].

Network structure presentation should be the one which assists human network manager to have a better overview on the network, which just displays the topologic structure of the network as network graph on the screen such that manager could refer to this and analyze what goes wrong within this network or which part needs to be improved or repaired [4]. It could improve work efficiency of network manager, and there are several network corporations calling for this function, while this method seems scarce.

\section{Related Work}

Network structure description is the protagonist to show to managers; however it is inefficient that getting the useful information managers would spend a lot of time and energy on reading and analyzing. 
It will reduce this cost down greatly if we demonstrate all those network properties as a network graph clearly on a screen [5]. In this case, getting the network structure description in a specified form is prerequisite [6].

Network structure description is easy to obtain. In many cases, managers can store the descriptions in the specified form in this case. However, in other cases, network structure may change greatly in a short period; it is not possible to keep the description about these networks. In this case, they are still able to get the description; although it is not our concern in this paper.

\section{Our Approach}

\subsection{Related Concepts}

\section{1) Crossing}

A parameter is in need which measures a picture whether it is clear enough. It could take the quantity of the crossings in the network graph as the parameter for clear score.

2) Valid range

Obviously, not only do crossings lead graphs look bad, but also large range does. Therefore, graph should be small. The range is the smaller the better.

3) Balance degree

As the display range is a rectangle, it could be split into several little squares. Count the dots in each square and get the variance of them. The variance works as Balance degree. There is an available method given as follow to illustrate the balance degree.

4) Center circle

WAN is always built with a backbone, which usually presents as a circle to reduce distance between two peers, and called center circle in this paper. In some cases, there is no such a backbone; we could get a center part in graphics. It will be called center circle as well.

\subsection{Approach and model}

Step 1: Center circle getting

In many cases, the center circles are the backbones of the network. It is easy to find the center circles out. But in some cases, all the peers in the network are coequal. For example, IOV usually contains no backbone, but a center circle is necessary as well. Center circle should satisfy the requirement in graphics.

Center, in graphics, has a very elegant property that it keeps the minimal maximum of distance away to any part of this patterning. According to this, get these points with short distances between their furthest points. If these points constitute a loop, it is the center circle. If not, take more points satisfy the upper requirement, until they form a loop or they are too many and they are connected.

Step 2: Shortest tree

Just like the minimum spanning tree, the shortest tree was built by broad first search (BFS) to reduce the height of spanning tree. Every node in this tree owns the least distance from the root; the tree will be compact and well-balanced after printing on a screen.

As the root is center circle, polar coordinates space is helpful to the tree node arranging. Assume there is a super root which is the father of the nodes in center circle, and it is at origin. The super root will assign its free angle area to its children rationally, and the children inherit the area from their parent, and they will assign their own area to their children, too, until it has no child. Therefore, children should keep a proper distance from their parents after getting the areas from them.

In this model, there many variables could be change to adjust the model's performance. In this paper, we will take one to illustrate.

Some parameters, just like distance, in this algorithm could be changed to any reasonable value, without troubles cased 
by these changes.

Step 3: Gravitational evolution

According to the Newton's law of motion, the universe could not continue, if gravitation works alone, without some powers to counteract with gravitation [7]. Assume that all the peers in the network are mass point with same electric charge, and they will exclude and attract with each other caused by charge and mass respectively. They will achieve a balance eventually, and what should be done is giving them an ideal initial state and a rational balance between gravitation and electromagnetic force, the remaining is waiting the system to evolve.

The initial state could be built by the previous model, and the relationship may cost some time. As we just need a balance, it's not necessary to calculate the precise value of the gravitation and the Coulomb force. What should do is find a way to reconcile the two forces, and now, there is an obvious inconsistent that the two forces keep a negative correlation with the distance similarly, however they have opposite directions. For example, we could take a formula from Hooke's law according to which the force will increase linearly with the distance rise, instead of the real gravitation. In fact, the relationship between two forces depends on the ideal gap.

The evolution will be an endless iteration process without external interrupt, which makes this very time-consuming. It is a nice way to optimize the graph autonomously, and able to lead a good result randomly, although it costs a lot of computing power.

\section{Experiments}

\subsection{Data set}

The data set used in our experiment consists of the network data provided by $\mathrm{He}$ nan department of China Mobile, which are extracted from the networks in use.
We take about 100 samples to test the performance of our approach. In order to make the data set more varied, we tried our best to get networks different area and levels.

\subsection{Effect metrics}

The effect is measured hardly because of its subjectivity. Showing to people may be the best way to measure the effect of demonstrating. We just take a sample randomly to show in this paper, because of the length of an article.

There are about 170 nodes, and 190 links in this sample. Several renderings produced by different method are showed to compare as follow.

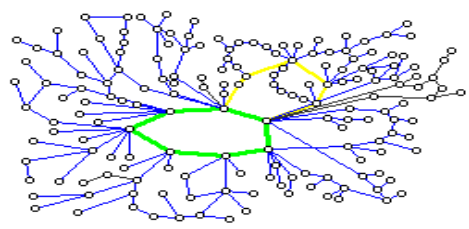

Fig. 1: Manual Trim

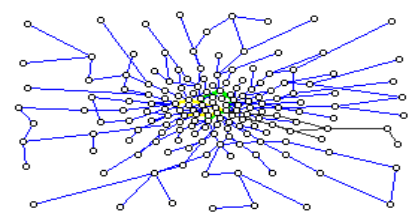

Fig. 2: Graphviz®

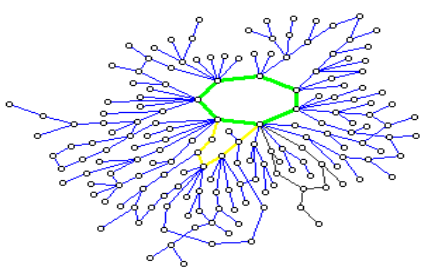

Fig. 3: Shortest tree

In Fig. 1, 2 and 3, we can see that the ways in our paper keep obvious advantages to traditional methods.

\subsection{Performance metrics}

The parameters defined in advance should be measure to compare with the traditional methods. 
$\mathrm{L}$ and $\mathrm{N}$ stands for the set of links and nodes in the network. In this part,

Crossing keeps a simple formula as its definition as follow.

$$
\begin{gathered}
\text { Cross }=\sum_{i, j \in L} f_{\text {cross }}(i, j) \\
f_{\text {cross }}(i, j)=\left\{\begin{array}{lc}
1, & i, j \text { cross } \\
0, & \text { else }
\end{array}\right.
\end{gathered}
$$

Valid range is two-tuples which easy to get, and described as follow.

$$
V R=\left[\max _{i, j \in N}|x(i)-x(j)|, \max _{i, j \in N}|y(i)-y(j)|\right]
$$

The formula of consumed time and error rate will not give out.

Two traditional methods, manual trim and Graphviz ${ }^{\circledR}$, were measured in our experiments, although it is not professional for network demonstrating. Each model mentioned above was tested singly in our experiments, and a compounded one.

\subsection{Results}

\begin{tabular}{|l|l|l|l|l|l|}
\hline & crossing & valid range & consumed time & error rate & balance degree \\
\hline Manual trim & 0.08 & {$[957,710]$} & $1.5 \sim 2.5$ hours & $5 \sim 7 \%$ & 10.579 \\
\hline Graphviz ${ }^{\circledR}$ & 0.02 & {$[20000+, 18000+]$} & 32.50 seconds & $0 \%$ & 183.29 \\
\hline Shortest tree & 0.02 & {$[459,450]$} & $>1.65$ seconds & $0 \%$ & 20.531 \\
\hline
\end{tabular}

\section{Conclusions}

In this paper, we proposed a network structure presentation approach. In our approach, we designed an algorithm system called shortest tree and realize an auxiliary model to adjust the demonstration. Next, we improve the visual effectiveness by auxiliary model to obtain an acceptable result. Experimental results tested from our data set showed that this novel approach was effective and robust compared to traditional approaches.

In our future work, we will improve our approach to make it applicable to other kinds of networks. We also plan to adopt our approach for network structure optimizing applications.

\section{Acknowledgement}

This paper is supported by National Natural Science Foundation of China No.61272512 and Beijing Municipal Natural Science Foundation No.4121001.

\section{References}

[1] Computer-aided

Network
Management - GFL Dixon - Electric Energy Conference 1980

[2] Large-scale Network Layout Optimization for Radial Distribution Networks by Parallel Computing JC Moreira, E Miguez, C Vilacha Power Delivery, IEEE, 2011

[3] Design and Implementation of Automatic Topology Discovery for EPON_EOC Unified Management System - Z Pan, J Nie, W Liu, H Huang - Energy Procedia, 2011

[4] Design of the Network Topology Display Module in SNMP-Based GPON Network Management System - S Chen, JX Zhang - Jisuanji Xitong Yingyong- Computer Systems \& Applications, 2011

[5] Network Management Requirements for MPLS-based Transport Networks - K Lam, S Mansfield, E Gray - 2010

[6] Topology Optimization: Theory, Methods and Applications. MP Bendsoe, O Sigmund - 2004 Immunity-Based Gravitational Search Algorithm. Yu Zhang, Yana Li, Feng Xia, Ziqiang Luo. Information Computing and Applications. Lecture Notes in Computer Science Volume 7473, 2012, pp 754-761 